TRANSACTIONS OF THE

AMERICAN MATHEMATICAL SOCIETY

Volume 356, Number 12, Pages 4857-4872

S 0002-9947(04)03396-3

Article electronically published on January 13, 2004

\title{
ON ORBITAL PARTITIONS AND EXCEPTIONALITY OF PRIMITIVE PERMUTATION GROUPS
}

\author{
R. M. GURALNICK, CAI HENG LI, CHERYL E. PRAEGER, AND J. SAXL
}

\begin{abstract}
Let $G$ and $X$ be transitive permutation groups on a set $\Omega$ such that $G$ is a normal subgroup of $X$. The overgroup $X$ induces a natural action on the set $\operatorname{Orbl}(G, \Omega)$ of non-trivial orbitals of $G$ on $\Omega$. In the study of Galois groups of exceptional covers of curves, one is led to characterizing the triples $(G, X, \Omega)$ where $X$ fixes no elements of $\operatorname{Orbl}(G, \Omega)$; such triples are called exceptional. In the study of homogeneous factorizations of complete graphs, one is led to characterizing quadruples $(G, X, \Omega, \mathcal{P})$ where $\mathcal{P}$ is a partition of $\operatorname{Orbl}(G, \Omega)$ such that $X$ is transitive on $\mathcal{P}$; such a quadruple is called a $T O D$ (transitive orbital decomposition). It follows easily that the triple $(G, X, \Omega)$ in a TOD $(G, X, \Omega, \mathcal{P})$ is exceptional; conversely if an exceptional triple $(G, X, \Omega)$ is such that $X / G$ is cyclic of prime-power order, then there exists a partition $\mathcal{P}$ of $\operatorname{Orbl}(G, \Omega)$ such that $(G, X, \Omega, \mathcal{P})$ is a TOD. This paper characterizes TODs $(G, X, \Omega, \mathcal{P})$ such that $X^{\Omega}$ is primitive and $X / G$ is cyclic of primepower order. An application is given to the classification of self-complementary vertex-transitive graphs.
\end{abstract}

\section{INTRODUCTION}

Given a transitive permutation group $G$ on a set $\Omega$, there is a natural action of $G$ induced on the set $\Omega \times \Omega$. The $G$-orbits in this action are the $G$-orbitals on $\Omega$. The trivial orbital is the diagonal orbit $\Delta=\{(\omega, \omega) \mid \omega \in \Omega\}$; the other orbitals are said to be non-trivial. We write $\Omega^{(2)}:=(\Omega \times \Omega) \backslash \Delta$, and denote by $\operatorname{Orbl}(G, \Omega)$ the set of $G$-orbits in $\Omega^{(2)}$. A $G$-orbital decomposition is a partition of $\operatorname{Orbl}(G, \Omega)$, which induces a $G$-invariant partition of $\Omega^{(2)}$, and the number of parts is called the index of the decomposition. If a $G$-orbital decomposition $\mathcal{P}$ and a group $X$ with $G<X \leq \operatorname{Sym}(\Omega)$ are such that $\mathcal{P}$ is $X$-invariant and $X$ is transitive on $\mathcal{P}$, then the quadruple $(G, X, \Omega, \mathcal{P})$ is called a transitive orbital decomposition, or a TOD for short. A TOD $(G, X, \Omega, \mathcal{P})$ is said to be cyclic if $X^{\mathcal{P}}$ is cyclic.

We observe that if $(G, X, \Omega, \mathcal{P})$ is a TOD of index $k$, then so is $(\hat{G}, X, \Omega, \mathcal{P})$, where $\hat{G}$ is the kernel of $X$ acting on $\mathcal{P}$. Now $G \leq \hat{G}$ and $\hat{G}$ is normal in $X$. Thus, replacing $G$ by $\hat{G}$, we may always replace a cyclic TOD $(G, X, \Omega, \mathcal{P})$ by $(\hat{G}, X, \Omega, \mathcal{P})$, which is a cyclic TOD with $\hat{G}$ normal in $X$ and $X / \hat{G}$ is a cyclic group.

Received by the editors October 5, 2002 and, in revised form, April 15, 2003.

2000 Mathematics Subject Classification. Primary 20B15, 20B30, 05C25.

This paper is part of a project funded by the Australian Research Council. The first author acknowledges support from NSF grant DMS 0140578, and the first and fourth authors acknowledge support by an EPSRC grant. 
Transitive orbital decompositions arise in a number of different areas. The reader is referred to [LP01] for further motivation and background. For example, the case where $k=2$ corresponds to a class of digraphs which is important to Ramsey numbers: For a digraph $\Gamma$ with vertex set $V$, the complement $\bar{\Gamma}$ of $\Gamma$ is the digraph with vertex set $V$ such that $\left(v, v^{\prime}\right)$ is an edge of $\bar{\Gamma}$ if and only if it is not an edge of $\Gamma$. In the case where $\Gamma$ is isomorphic to $\bar{\Gamma}$, the digraph $\Gamma$ is said to be self-complementary. Then a TOD of index 2 exactly corresponds to a self-complementary vertex-transitive digraph. The class of self-complementary vertex-transitive digraphs has received considerable attention in the literature, see for example Li97, [LP00, Muz99], Sac62. We now recall the concept of exceptional permutation groups. Let $G$ be a transitive normal subgroup of a permutation group $X$ on $\Omega$. Then $X$ induces an action on $\operatorname{Orbl}(G, \Omega)$. If $X$ fixes no element in $\operatorname{Orbl}(G, \Omega)$, then the triple $(G, X, \Omega)$ is said to be exceptional. The exceptionality of transitive permutation groups arises naturally in considerations of Galois groups of exceptional covers of curves - cf. [FGS93], GMS01]. Much information on exceptional triples may be found in [GMS01.

By definition, if $(G, X, \Omega, \mathcal{P})$ is a cyclic TOD with $G \triangleleft X$ and $X / G=X^{\mathcal{P}}$, then $(G, X, \Omega)$ is an exceptional triple. On the other hand, an exceptional triple $(G, X, \Omega)$ with $X / G$ cyclic of prime-power order gives rise to a cyclic TOD $(G, X, \Omega, \mathcal{P})$ of prime-power index, see Lemma 2.1. Cyclic TODs of prime index play a central role in the study of TODs $(G, X, \Omega, \mathcal{P})$ : Since $X$ acts transitively on $\mathcal{P}$ of degree $k$, by a theorem of [FKS81], $X$ contains an element $x$ of order $p^{e}$ with $p$ a prime dividing $k$ and $e \geq 1$, which acts fixed-point-freely on $\mathcal{P}$; it then follows that there is a partition $\mathcal{Q}$ of index $p$ refined by $\mathcal{P}$ such that $(G,\langle G, x\rangle, \Omega, \mathcal{Q})$ is a TOD of index $p$ (cf. [LP01, Lemma 3.6]). A TOD $(G, X, \Omega, \mathcal{P})$ is said to be point-primitive if $X$ is primitive on $\Omega$. It is shown in [LP01, Theorem 1.2] that to each TOD $(G, X, \Omega, \mathcal{P})$ there corresponds at least one point-primitive TOD $(\hat{G}, \hat{X}, \hat{\Omega}, \hat{\mathcal{P}})$ of the same index with $\hat{G}, \hat{X}$ sections of $G, X$ respectively, $\hat{\Omega} \subset \Omega$ and $X^{\mathcal{P}}=\hat{X}^{\hat{\mathcal{P}}}$. This suggests that an understanding of point-primitive TODs is crucial for a proper understanding of TODs in general.

The product of all minimal normal subgroups of a group $G$ is called the socle of $G$ and denoted by $\operatorname{soc}(G)$. A group $X$ is said to be almost simple if its socle $T$ is a nonabelian simple group; we will always identify such a group $X$ with a subgroup of $\operatorname{Aut}(T)$. We develop a result of [GMS01] to obtain the following classification.

Theorem 1.1. Let $(G, X, \Omega, \mathcal{P})$ be a TOD with $X$ an almost simple group primitive on $\Omega, G$ normal in $X$, and $X / G$ a cyclic p-group for some prime $p$. Let $H$ denote the stabilizer in $X$ of a point in $\Omega$ and set $T=\operatorname{soc}(X)$. Let $\sigma$ be an outer automorphism of $G$ such that $X=\langle G, \sigma\rangle$. Then one of the following holds, and in each case we get examples:

(i) $T=L\left(q^{p}\right)$ is a simple Chevalley group defined over a field of order $q^{p}, \sigma$ is a field automorphism, $H$ is the normalizer of $L(q)$ and $p$ does not divide the order of $L\left(q^{p}\right)$;

(ii) $T=\mathrm{PSL}_{2}\left(2^{e}\right)$ with $e$ a power of $p$ and $p$ odd, $\sigma$ is a field automorphism of order $e$ and $H \cap T \cong \mathrm{D}_{2\left(2^{e}+1\right)}$ is the normalizer of a nonsplit torus of $T$;

(iii) $T=\mathrm{PSL}_{2}\left(q^{e}\right)$ with e even, $q$ odd, $p=2, G$ is an extension of odd index of $T, \sigma$ is an involutory field-diagonal automorphism and $H \cap T \cong \mathrm{D}_{q^{e}-1}$ is the normalizer of a split torus of $T$; 
(iv) $T=\mathrm{PSU}_{3}\left(2^{e}\right)$ with $p=3$ and $e>1$, $e$ a power of $3, \sigma$ is a field-diagonal automorphism of order $e$ and $H \cap T$ is the stabilizer of an orthogonal decomposition of the natural module;

(v) $T=\mathrm{Sz}\left(2^{e}\right)$, e a power of an odd prime $p$ other than $5, \sigma$ is a field automorphism of order $e$, and $H \cap T$ is the normalizer of a Sylow 5-subgroup of $T$.

Let $X$ be a transitive permutation group on a set $\Omega$, and let $N=\operatorname{soc}(X)$. For a subgroup $Y \leq X$, denote by $Y_{\omega}$ the stabilizer in $Y$ of $\omega \in \Omega$, that is, $Y_{\omega}=\left\{y \in Y \mid \omega^{y}=\omega\right\}$. Assume that $N$ is transitive on $\Omega$. If $N$ is an elementary abelian group, then $X$ is said to be of affine type. Suppose now that $N=T^{l}$ is a minimal normal subgroup of $X$ and that $T$ is a nonabelian simple group. If $N_{\omega} \cong T$, then $X$ is said to be of simple diagonal type; if $N_{\omega} \cong T^{m}$ for some integer $m \geq 2$, then $X$ is said to be of compound diagonal type; while if $1 \neq N_{\omega} \neq T^{m}$ for any $m \geq 1$ and $l \geq 2$, then $X$ is said to be of product action type. See Section 2 for more details of these types.

The next theorem gives a characterization of point-primitive cyclic TODs of prime-power index.

Theorem 1.2. Let $(G, X, \Omega, \mathcal{P})$ be a cyclic TOD of index $p^{e}$ with $p$ prime and $X$ primitive on $\Omega$, and let $\operatorname{soc}(X)=T^{l}$, where $T$ is simple and $l \geq 1$. Then one of the following holds:

(i) affine: $T=\mathbb{Z}_{r}$ with $r$ prime and $r \neq p$;

(ii) almost simple: $T$ is nonabelian, and $l=1$ with $T, p$ as in Theorem 1.1;

(iii) simple diagonal: $T$ is nonabelian, $l=p^{e} \geq p$, and $p \nmid|T|$, as in Construction 2.5.

(iv) product action: $T$ is nonabelian, $l \geq 2, X \leq \operatorname{Aut}(T)<\mathrm{S}_{l}$ in product action on $\Omega=\Delta^{l}$, and there exists a point-primitive $T O D\left(T,\left\langle T, \sigma_{0}\right\rangle, \bar{\Delta}, \mathcal{Q}\right)$ of index $p^{e_{0}}$ for some positive integer $e_{0}$ as in part (ii) for some $T$-invariant partition $\bar{\Delta}$ of $\Delta$;

(v) compound diagonal: $T$ is nonabelian, $l \geq 4, X$ is of compound diagonal type on $\Delta^{l_{0}}$ with $l_{0} \geq 2, l_{0} \mid l$, and there exists a TOD $\left(T,\left\langle T, \sigma_{0}\right\rangle, \bar{\Delta}, \mathcal{Q}\right)$ of index $p^{e_{0}}$ for some positive integer $e_{0}$ as in part (iii) for some $T^{l / l_{0}}$ invariant partition $\bar{\Delta}$ of $\Delta$.

Moreover, in each case there exist examples.

Remark. The almost simple examples are classified in Theorem 1.1] and Construction 2.5 gives all simple diagonal examples. For any almost simple or simple diagonal example, and any $l$ or $l_{0}$, Construction 2.8 or 2.9 gives an example of product action type or compound diagonal type respectively.

We now turn our attention to the case of self-complementary graphs. For a TOD $(G, X, \Omega, \mathcal{P})$ of index $k$ with $\mathcal{P}=\left\{P_{1}, \ldots, P_{k}\right\}$, there are $k$ digraphs $\Gamma_{i}$ with vertex set $\Omega$ and edge set $P_{i}$. They form a homogeneous factorization of the complete digraph $\mathrm{K}_{n}$ where $n=|\Omega|$. As observed before, in the case where $k=2$, the factor $\Gamma_{2}=\bar{\Gamma}_{1}$, and $\Gamma_{1}$ is a self-complementary digraph. As a corollary of Theorem 1.2 we have a characterization of self-complementary digraphs with $X$ primitive on vertices. In [LP00], there is a construction of the first family of self-complementary digraphs which are vertex-transitive but not Cayley digraphs. Many of the examples given are undirected, that is, if $\left(\omega, \omega^{\prime}\right)$ is an edge then so is $\left(\omega^{\prime}, \omega\right)$. However, 
the initial digraphs used in the construction in [LP00], with $q^{2}\left(q^{2}+1\right) / 2$ vertices and automorphism group $\operatorname{PSL}_{2}\left(q^{2}\right)$, are not undirected. A question was left open as to whether the initial digraphs could have been chosen to be undirected. We now answer this by showing that there are no such undirected graphs with almost simple automorphism groups. For a self-complementary digraph $\Gamma$, an isomorphism between $\Gamma$ and $\bar{\Gamma}$ is called a complementing permutation.

Theorem 1.3. Let $\Gamma$ be a vertex-transitive self-complementary digraph with vertex set $V$. Let $G=$ Aut $\Gamma$, and let $\sigma$ be a complementing permutation. Assume that $X:=\langle G, \sigma\rangle$ is primitive on $V$. Then one of the following is true:

(i) $X$ is an affine group with socle of odd order;

(ii) $X$ is almost simple with socle $\mathrm{PSL}_{2}\left(q^{2}\right)$ with $q$ odd;

(iii) $X$ is of product action type with socle $\mathrm{PSL}_{2}\left(q^{2}\right)^{l}$, where $q$ is odd and $l \geq 2$.

Moreover, if $\Gamma$ is undirected, then $X$ is affine or of product action type.

For the almost simple case, we further have

Corollary 1.4. Let $\Gamma$ be a vertex-transitive self-complementary digraph with vertex set $V$ and $\sigma$ a complementing permutation. Assume that Aut $\Gamma$ is almost simple. Then

(i) $\Gamma$ is not undirected;

(ii) $|V|=q^{2}\left(q^{2}+1\right) / 2, \operatorname{soc}(\mathrm{Aut} \Gamma)=\mathrm{PSL}_{2}\left(q^{2}\right)$ with $q$ odd, and $\sigma$ is a fielddiagonal automorphism.

\section{Constructing examples}

Let $G$ be a transitive permutation group on a set $\Omega$. For $\omega \in \Omega$, an orbit of $G_{\omega}$ on $\Omega \backslash\{\omega\}$ is called a suborbit of $G$ at $\omega$. Orbitals and suborbits at $\omega$ are in one-to-one correspondence: an orbital $\Delta$ uniquely corresponds to a suborbit $\Delta(\omega)=\left\{\omega^{\prime} \mid\left(\omega, \omega^{\prime}\right) \in \Delta\right\}$ at $\omega$. Identify $\Omega$ with $\left[G: G_{\omega}\right]=\left\{G_{\omega} g \mid g \in G\right\}$, and identify the action of $G$ on $\Omega$ with the coset action of $G$ on $\left[G: G_{\omega}\right]$. Then also suborbits of $G$ at $\omega$ exactly correspond to double cosets $G_{\omega} g G_{\omega}$, where $g \in G$.

2.1. A method for constructing cyclic TODs. For a transitive permutation group $G$ on a set $\Omega$, assume that $X$ is such that $G \triangleleft X \leq \operatorname{Sym}(\Omega)$ and $X / G$ is cyclic. Since $G$ is transitive, $X=G X_{\omega}$ and hence there exists an element $\sigma \in X_{\omega} \backslash G_{\omega}$ such that $X=\langle G, \sigma\rangle$. Since $G$ is normal in $X$, the subgroup $\langle\sigma\rangle$ induces an action on the double cosets of $G_{\omega}$ by

$$
\left(G_{\omega} g G_{\omega}\right)^{\sigma}=G_{\omega} g^{\sigma} G_{\omega}, \text { where } g \in G .
$$

Through this action, $\langle\sigma\rangle$ acts on $\operatorname{Orbl}(G, \Omega)$. The following lemma is an immediate consequence of [LP01, Lemma 6.1]; a similar result was proved in [GMS01].

Lemma 2.1. Let $G$ be a transitive permutation group on $\Omega$, and assume that $G \triangleleft X \leq \operatorname{Sym}(\Omega)$ is such that $X=\langle G, \sigma\rangle$, where $\sigma \in X_{\omega} \backslash G_{\omega}$ for some $\omega \in \Omega$. Assume further that $\sigma$ has prime-power order. Then the following are equivalent:

(a) there exists a partition $\mathcal{P}$ of $\Omega^{(2)}$ such that $(G, X, \Omega, \mathcal{P})$ is a cyclic TOD of index $k>1$ for some $k$ dividing $o(\sigma)$;

(b) $\sigma$ fixes no $G_{\omega} g G_{\omega}$ for $g \in G \backslash G_{\omega}$;

(c) $(G, X, \Omega)$ is an exceptional triple. 
We note that the element $\sigma$ in Lemma 2.1 induces a non-trivial automorphism of the group $G$ and normalizes the point-stabilizer $G_{\omega}$. By Lemma 2.1, in order to construct cyclic TODs of prime-power index, we only need to find groups $G$ with a core-free subgroup $H$ and an automorphism $\sigma$ such that $\sigma$ is of prime-power order, normalizes $H$, and fixes no double coset $H g H$ for $g \in G \backslash H$. A method for constructing cyclic TODs may be formulated as follows. This is a special case of [LP01, Construction 3.1].

Construction 2.2. Assume that a group $G$ has a core-free subgroup $H$ and an automorphism $\sigma$ such that $\sigma$ has p-power order with $p$ prime and $\sigma$ normalizes $H$. Assume further that $\sigma$ fixes no $H g H$, where $g \in G \backslash H$. Let $X=\langle G, \sigma\rangle$, and let $\Omega=[G: H]$. Let $\Delta_{1}, \ldots, \Delta_{r}$ be the orbits of $\langle\sigma\rangle$ acting on $\operatorname{Orbl}(G, \Omega)$, and let $l_{i}=\left|\Delta_{i}\right|$. Clearly, each $l_{i}$ is a $p$-power. Let $k=\min \left\{l_{1}, \ldots, l_{r}\right\}$, and let $\Delta_{i 1}, \ldots, \Delta_{i k}$ be the orbits of $\left\langle\sigma^{k}\right\rangle$ acting on $\Delta_{i}$. Let $P_{j}=\bigcup_{1 \leq i \leq r} \Delta_{i j}$, and let $\mathcal{P}=\left\{P_{1}, \ldots, P_{k}\right\}$. Then $(G, X, \Omega, \mathcal{P})$ is a cyclic TOD of index $k$.

It is easily shown that $\mathcal{P}$ is a partition of index $k$ of $\operatorname{Orbl}(G, \Omega)$ such that $\langle\sigma\rangle$ is transitive on $\mathcal{P}$, and thus the quadruple $(G, X, \Omega, \mathcal{P})$ given by Construction 2.2 is a cyclic TOD of index $k$, and the triple $(G, X, \Omega)$ is an exceptional triple.

It is well known that a group with a fixed-point-free automorphism is soluble, see [GO, Theorem 1.48] or [GMS01, Proposition 3.22]. This, together with a result of [FKS81] and Lemma [2.1, yields the next lemma, see [GMS01, 3.23] or [LP01. Lemma 3.7].

Lemma 2.3. Let $(G, X, \Omega, \mathcal{P})$ be a TOD of index $k$ such that $X$ has a regular normal subgroup $N$ contained in $G$. Then $N$ is soluble.

This shows that if $(G, X, \Omega, \mathcal{P})$ is a point-primitive TOD and $X$ has a normal subgroup which is regular on $\Omega$, then $X$ is an affine group, that is, $\operatorname{soc}(X)$ is elementary abelian. There are many known examples of TODs of this type, see for instance [LP01, Construction 6.3]. Now we assume that $(G, X, \Omega, \mathcal{P})$ is a pointprimitive TOD and $\operatorname{soc}(X)$ is nonabelian. Then by Lemma $2.3 \operatorname{soc}(X)$ is not regular, and thus by the O'Nan-Scott theorem (refer to [LPS00]), either $X$ is almost simple, or $X$ is one of the types: simple diagonal, compound diagonal or product action. In the rest of this section, we will construct cyclic TODs corresponding to each of these four types of primitive permutation groups.

2.2. Almost simple case. There are five infinite families of almost simple groups giving rise to TODs. In each of the following cases, we have a triple $(G, H, \sigma)$ such that $(G,\langle G, \sigma\rangle, \Omega)$ is exceptional where $\Omega=[G: H]$ and $\sigma \in \mathbf{N}_{\operatorname{Sym}(\Omega)}(H)$ :

(i) Let $G=L\left(q^{p}\right)$ be a simple Chevalley group defined over a field of order $q^{p}$ with $p$ a prime that does not divide the order of $L(q)$. Let $H$ be the normalizer of $L(q)$ in $G$, and let $\sigma$ be a field automorphism of $G$ of order a power of $p$.

(ii) Let $G=\operatorname{PSL}_{2}\left(2^{e}\right)$, where $e$ is a $p$-power with $p$ an odd prime. Let $\sigma$ be a field automorphism of $G$ of order $e$, and let $H \cong \mathrm{D}_{2\left(2^{e}+1\right)}$ be the normalizer of a nonsplit torus of $G$.

(iii) Let $G=\mathrm{PSL}_{2}\left(q^{2}\right)$ with $q$ odd, and let $H$ be the normalizer of a split torus isomorphic to $\mathrm{D}_{q^{2}-1}$. Let $\sigma$ be the product of a diagonal automorphism and a field automorphism of even order. 
(iv) Let $G=\operatorname{PSU}_{3}\left(2^{e}\right)$ be such that $e$ is a power of 3 , and let $H$ be the stabilizer of an orthogonal decomposition of the natural module. Let $\sigma$ be a fielddiagonal automorphism of order $e$.

(v) Let $G=\mathrm{Sz}\left(2^{e}\right)$ be such that $e$ is a power of the odd prime $p \neq 5$, and let $H$ be the normalizer in $G$ of a Sylow 5-subgroup. Let $\sigma$ be a field automorphism of order $e$.

The next lemma, proved in [GMS01, §3], tells us that each triple $(G, H, \sigma)$ given above gives rise to an exceptional triple.

Lemma 2.4. Let $(G, H, \sigma)$ be one of the triples listed above. Then $\sigma$ fixes no $\mathrm{HgH}$ for $g \in G \backslash H$, that is, $(G,\langle G, \sigma\rangle,[G: H])$ is an exceptional triple.

Thus by Construction 2.2, there exists a partition $\mathcal{P}$ of $\operatorname{Orbl}(G, \Omega)$ such that $(G, X, \Omega, \mathcal{P})$ is a cyclic TOD.

2.3. Simple diagonal construction. A permutation group is said to be quasiprimitive if all of its non-trivial normal subgroups are transitive. A TOD $(G, X, \Omega, \mathcal{P})$ is said to be point-quasiprimitive if $X$ is quasiprimitive on $\Omega$.

The following construction produces triples $(G, X, \sigma)$ with $X$ quasiprimitive of simple diagonal type, and implies that there are many cyclic TODs of this type.

Construction 2.5. (This constructs a triple $(G, H, \sigma)$ such that $(G,\langle G, \sigma\rangle, \Omega)$ is exceptional, where $\Omega=[G: H]$.) Let $G=T_{1} \times \cdots \times T_{p^{e}} \cong T^{p^{e}}$, where $p$ is a prime and $T_{1} \cong \ldots \cong T_{p^{e}} \cong T$, where $T$ is a nonabelian simple group such that $p \nmid|T|$. Let $H=\{(t, t, \ldots, t) \mid t \in T\}$, a full diagonal subgroup of $G$. Let $\sigma$ be the automorphism of $G$ of order $p^{e}$ defined by

$$
\sigma:\left(t_{1}, t_{2}, \ldots, t_{p^{e}}\right) \rightarrow\left(t_{p^{e}}, t_{1}, t_{2}, \ldots, t_{p^{e}-1}\right)
$$

for $\left(t_{1}, \ldots, t_{p^{e}}\right) \in G$. Then $\langle\sigma\rangle$ acts cyclically on the factors $T_{1}, T_{2}, \ldots, T_{p^{e}}$, and $\sigma$ normalizes $H$. Let $\Omega=[G: H]$, and let $X=\langle G, \sigma\rangle$. Then $X<G$. $\left(\operatorname{Out}(T) \times \mathrm{S}_{p^{r}}\right)<$ $\operatorname{Sym}(\Omega)$, and in this representation $\sigma=\left(12 \ldots p^{r}\right) \in \mathrm{S}_{p^{r}}$.

The following lemma shows that a triple $(G, X, \sigma)$ produced in Construction 2.5 gives rise to a cyclic TOD.

Lemma 2.6. Let $(G, X, \sigma)$ be as in Construction 2.5. Then $\sigma$ fixes no $\mathrm{HgH}$ for $g \in G \backslash H$, so $(G, X,[G: H])$ is an exceptional triple.

Proof. Suppose that an element $g=\left(t_{1}, t_{2}, \ldots, t_{p^{e}}\right) \in G$ is such that $\sigma$ fixes $H g H$, that is, $g^{\sigma}=h_{1} g h_{2}$ for some $h_{1}, h_{2} \in H$. We have that $h_{1}=(x, x, \ldots, x)$ and $h_{2}=(y, y, \ldots, y)$ with $x, y \in T$. Then

$$
\begin{aligned}
\left(t_{p^{e}}, t_{1}, \ldots, t_{p^{e}-1}\right)=g^{\sigma} & =h_{1} g h_{2} \\
& =(x, x, \ldots, x)\left(t_{1}, t_{2}, \ldots, t_{p^{e}}\right)(y, y, \ldots, y) \\
& =\left(x t_{1} y, x t_{2} y, \ldots, x t_{p^{e}} y\right) .
\end{aligned}
$$

Writing $s=t_{p^{e}}$, we have that $t_{p^{e}-i}=x^{i} s y^{i}$ for $i=1,2, \ldots, p^{e}$ (writing $t_{0}=t_{p^{e}}$ ). In particular, $s=x^{p^{e}} s y^{p^{e}}$. It follows that $s=x^{p^{e} k} s y^{p^{e} k}$ for all integers $k$ (positive and negative). Since $p$ is coprime to $|T|$, there exists an integer $j$ such that $p^{e} j \equiv 1$ $(\bmod |T|)$, and so $x^{p^{e} j}=x$ and $y^{p^{e} j}=y$. Hence $s=x^{p^{e} j} s y^{p^{e} j}=x s y$, and so $s=x^{i} s y^{i}$ for all $i$. Thus $t_{1}=t_{2}=\cdots=t_{p^{e}}=s$, and so $g \in H$. 
2.4. Product action construction. Here we provide general constructions that take as input a triple $(G, K, \sigma)$ from Subsection 2.2 or 2.3 and an integer $l \geq 2$, and produce an exceptional triple for the group $G^{l}$. These constructions make use of the product action of a wreath product. A crucial result needed for these constructions is proved in Lemma 2.7

Let $G=N_{1} \times N_{2} \times \cdots \times N_{l}$ be such that $N_{1} \cong N_{2} \cong \cdots \cong N_{l} \cong N$. Then Aut $(G)$ has a subgroup Aut $(N)<\mathrm{S}_{l}$. Assume that $\sigma$ is an element of $\operatorname{Aut}(N)<\mathrm{S}_{l}$ such that $N_{i}^{\sigma}=N_{i+1}$ for each $i$ (reading the subscripts modulo $l$ ). Then $\sigma^{l}$ normalizes each $N_{i}$. Suppose that $K=H_{1} \times H_{2} \times \cdots \times H_{l}$ is a subgroup of $G$ such that $H_{i}<N_{i}, \sigma$ normalizes $K$ and $\sigma^{l}$ normalizes each $H_{i}$. Then $\langle\sigma\rangle$ acts naturally on $\{K g K \mid g \in G \backslash K\}$ and $\left\langle\sigma^{l}\right\rangle$ acts naturally on $\left\{H_{i} t H_{i} \mid t \in N_{i} \backslash H_{i}\right\}$ for each $i$. Moreover, we have

Lemma 2.7. Let $G, K, \sigma$ be as above. Then $\sigma$ fixes no $K g K$ for $g \in G \backslash K$ if and only if for all $i, \sigma^{l}$ fixes no $H_{i} t H_{i}$ with $t \in N_{i} \backslash H_{i}$.

Proof. Suppose that $\sigma$ fixes $K g K$ for some $g \in G \backslash K$, that is, $K g^{\sigma} K=(K g K)^{\sigma}=$ $K g K$. Then in particular, $K g^{\sigma^{l}} K=(K g K)^{\sigma^{l}}=K g K$. Write $g=\left(t_{1}, t_{2}, \ldots, t_{l}\right) \in$ $G$, where $t_{i} \in N_{i}$. Then we have

$$
\begin{aligned}
& K g K=\left(H_{1} t_{1} H_{1}\right) \times\left(H_{2} t_{2} H_{2}\right) \times \cdots \times\left(H_{l} t_{l} H_{l}\right), \text { and } \\
& K g^{\sigma^{l}} K=(K g K)^{\sigma^{l}}=\left(H_{1} t_{1}^{\sigma^{l}} H_{1}\right) \times\left(H_{2} t_{2}^{\sigma^{l}} H_{2}\right) \times \cdots \times\left(H_{l} t_{l}^{\sigma^{l}} H_{l}\right),
\end{aligned}
$$

so that $\left(H_{i} t_{i} H_{i}\right)^{\sigma^{l}}=H_{i} t_{i}^{\sigma^{l}} H_{i}=H_{i} t_{i} H_{i}$ for each $i$. Since $g \notin K$, there exists $i$ such that $t_{i} \notin H_{i}$, and hence $\sigma^{l}$ fixes $H_{i} t_{i} H_{i}$ with $t_{i} \notin N_{i} \backslash H_{i}$.

Assume now that $\sigma^{l}$ fixes $H_{1} t H_{1}$ for some $t \in N_{1} \backslash H_{1}$, that is, $H_{1} t^{\sigma^{l}} H_{1}=$ $\left(H_{1} t H_{1}\right)^{\sigma^{l}}=H_{1} t H_{1}$. Then $t^{\sigma^{l}} \in H_{1} t H_{1}$, and so $t^{\sigma^{l}}=h_{1} t h_{2}$ for some $h_{1}, h_{2} \in H_{1}$. Now for $i=1, \ldots, l-1$, we have $t^{\sigma^{l+i}} \in N_{i+1}$. Let $g=\left(t^{\sigma^{l}}, t^{\sigma^{l+1}}, \ldots, t^{\sigma^{2 l-1}}\right)$. Then $g \in G \backslash K$, and

$$
\begin{aligned}
g^{\sigma} & =\left(t^{\sigma^{l}}, t^{\sigma^{l+1}}, \ldots, t^{\sigma^{2 l-1}}\right)^{\sigma} \\
& =\left(t^{\sigma^{2 l}}, t^{\sigma^{l+1}}, \ldots, t^{\sigma^{2 l-1}}\right) \\
& =\left(\left(h_{1} t h_{2}\right)^{\sigma^{l}}, t^{\sigma^{l+1}}, \ldots, t^{\sigma^{2 l-1}}\right) \\
& =\left(h_{1}^{\sigma^{l}} t^{\sigma^{l}} h_{2}^{\sigma^{l}}, t^{\sigma^{l+1}}, \ldots, t^{\sigma^{2 l-1}}\right) \\
& =\left(h_{1}^{\sigma^{l}}, 1, \ldots, 1\right)\left(t^{\sigma^{l}}, t^{\sigma^{l+1}}, \ldots, t^{\sigma^{2 l-1}}\right)\left(h_{2}^{\sigma^{l}}, 1, \ldots, 1\right) \in K g K .
\end{aligned}
$$

Thus $\sigma$ fixes $K g K$.

Point-quasiprimitive cyclic TODs of product action type and of compound diagonal type will be constructed below. As preparation we make the following definition of a certain type of transitive subgroup of a wreath product in product action. Let $N$ be a transitive permutation group on a set $\Delta$, and let $G=N_{1} \times \cdots \times N_{l}=N^{l}$ and $M=\mathbf{N}_{\text {Sym }(\Delta)}(N)$. For a subgroup $L \leq \mathrm{S}_{l}$, the product action of the wreath product $M \curlywedge L=M^{l} . L$ on $\Omega=\Delta^{l}$ is defined by

$$
\begin{aligned}
\left(x_{1}, \ldots, x_{l}\right): & \left(\delta_{1}, \ldots, \delta_{l}\right) \mapsto\left(\delta_{1}^{x_{1}}, \ldots, \delta_{l}^{x_{l}}\right), \\
\tau: & \left(\delta_{1}, \ldots, \delta_{l}\right) \mapsto\left(\delta_{1^{-1}}, \ldots, \delta_{l^{-1}}\right),
\end{aligned}
$$

for $\left(x_{1}, \ldots, x_{l}\right) \in M^{l}, \tau \in L$, and $\left(\delta_{1}, \ldots, \delta_{l}\right) \in \Omega=\Delta^{l}$. The subgroup $G$ is transitive on $\Omega$ since $N$ is transitive on $\Delta$, and $G$ is normal in $M$ L $L$ since $N$ is normal in $M$. Let $G<X<N<L$ be such that $X / G \cong \mathbb{Z}_{l}$ and $X$ permutes $N_{1}, \ldots, N_{l}$ transitively by conjugation. Let $\omega=(\delta, \ldots, \delta) \in \Omega$. Then we have $X=G X_{\omega}$, and 
hence there exists an element $\sigma \in X_{\omega} \backslash G_{\omega}$ such that $X=\langle G, \sigma\rangle$ and $X_{\omega}=\left\langle G_{\omega}, \sigma\right\rangle$. Let $H=N_{\delta}$, so that $G_{\omega}=H_{1} \times \cdots \times H_{l}=H^{l}$ and $(M \nmid L)_{\omega}=M_{\delta} \prec L$. Suppose now that $\sigma$ has the form $\sigma=(\psi, 1, \ldots, 1)(12 \ldots l)$, where $\psi \in \mathbf{N}_{M}(H)$. Then $\sigma^{l}=(\psi, \ldots, \psi)$, and $\langle N, \psi\rangle$ is a transitive permutation group on $\Delta$.

Construction 2.8. (This produces a triple $(G, K, \sigma)$ such that $(G,\langle G, \sigma\rangle, \Omega)$ is exceptional, where $\Omega=[G: K]$.) Let $N=T$ be a nonabelian simple group, and assume that $N$ is a transitive subgroup of $\operatorname{Sym}(\Delta)$. Let $H=N_{\delta}$ with $\delta \in \Delta$, and let $\psi \in \mathbf{N}_{\text {Sym }(\Delta)}(N) \cap \mathbf{N}_{\text {Sym }(\Delta)}(H)$ be such that $(N,\langle N, \psi\rangle, \psi)$ is a triple satisfying one of (i)-(iv) in Subsection 2.2. Let $G=N^{l}=N_{1} \times \cdots \times N_{l} \leq \operatorname{Sym}\left(\Delta^{l}\right)$, acting naturally on $\Omega=\Delta^{l}$. Let

$$
\begin{aligned}
& K=G_{(\delta, \ldots, \delta)}=H^{l}=H_{1} \times \cdots \times H_{l}, \\
& \Omega=[G: K], \text { and } \\
& \sigma=(\psi, 1, \ldots, 1)(12 \ldots l) \in\langle N, \psi\rangle \imath \mathrm{S}_{l}<\operatorname{Sym}(\Omega) .
\end{aligned}
$$

Construction 2.9. (This produces a triple $(G, K, \sigma)$ such that $(G,\langle G, \sigma\rangle, \Omega)$ is exceptional, where $\Omega=[G: K]$.) Let $N=T^{l_{0}}$, where $T$ is a nonabelian simple group and $l_{0}=p^{e}$ for some prime $p$ and $e \geq 1$ such that $p \nmid|T|$. Let $H=$ $\{(t, t, \ldots, t) \mid t \in T\}$ and $\Delta=[N: H]$, so that $N$ is a transitive subgroup of $\operatorname{Sym}(\Delta)$ and $H=N_{\delta}$ for $\delta=H \in \Delta$. Let $\psi \in \mathbf{N}_{\mathrm{Sym}(\Delta)}(N) \cap \mathbf{N}_{\mathrm{Sym}(\Delta)}(H)$ be such that $(N,\langle N, \psi\rangle, \psi)$ is a triple $(G, H, \sigma)$ produced in Construction 2.5. Let $G=N^{l}=N_{1} \times \cdots \times N_{l} \leq \operatorname{Sym}\left(\Delta^{l}\right)$, acting naturally on $\Omega=\Delta^{l}$. Let

$$
\begin{aligned}
& K=G_{(\delta, \ldots, \delta)}=H^{l}=H_{1} \times \cdots \times H_{l}, \\
& \Omega=[G: K], \text { and } \\
& \sigma=(\psi, 1, \ldots, 1)(12 \ldots l) \in \mathbf{N}_{\mathrm{Sym}(\Omega)}(G) .
\end{aligned}
$$

The following lemma shows that examples of point-quasiprimitive cyclic TODs of product action type and compound diagonal type may be produced by Constructions 2.8 and 2.9 .

Lemma 2.10. Any triple $(G,\langle G, \sigma\rangle, \Omega)$ produced by Construction 2.8 or Construction 2.9 is an exceptional triple.

Proof. Now $\sigma^{l}=(\psi, \psi, \ldots, \psi)$, and $\psi \in \mathbf{N}_{\mathrm{Sym}(\Delta)}(H)$. Since $(N,\langle N, \psi\rangle, \psi)$ is exceptional, by Lemma 2.1, $\sigma^{l}$ fixes no $H_{i} t H_{i}$ for $t \in N_{i} \backslash H_{i}$. Then by Lemma 2.7 $\sigma$ fixes no $K g K$ for $g \in G \backslash K$. Thus again by Lemma 2.1 $(G,\langle G, \sigma\rangle, \Omega)$ is an exceptional triple.

\section{Proof of Theorem 1.1}

The following lemma determines the point-primitive TODs of almost simple type, completing the proof of Theorem 1.1

Lemma 3.1. Let $(G, X, \Omega, \mathcal{P})$ be a cyclic point-primitive TOD such that $X / G$ is cyclic of p-power order with $p$ a prime. Assume further that $X$ is an almost simple group. Write $H=X_{\omega}$ for $\omega \in \Omega$, and let $T$ be the unique minimal normal subgroup of $X$. Let $\sigma$ be such that $X=\langle G, \sigma\rangle$. Then $T, \sigma, H$ satisfy one of (i)-(v) of Theorem 1.1. 
Proof. By Lemma 2.1, the triple $(G, X, \Omega)$ is exceptional. We use the classification theorems from [GMS01, Section 3]. We recall some of the argument there and refine it for our situation here. Arguing as in the first paragraph of Subsection 2.1, we get $X=\langle G, \sigma\rangle$ where $\sigma \in X_{\omega} \backslash G_{\omega}$ and we may take $\sigma$ to be a $p$-element.

If $T$ is sporadic or $\mathrm{A}_{n}$ with $n \neq 6$, there are no examples of exceptionality: this is not hard to show, see [GMS01, 3.34]. If $T=\mathrm{A}_{6}$, we view it as $\operatorname{PSL}_{2}(9)$ and it does occur in case (iii) of Theorem 1.1 with $G=T$.

Thus we may assume that $T=L\left(q_{0}\right)$ is a Lie type simple group defined over a field of order $q_{0}$, and $T$ is not isomorphic to an alternating group.

First suppose that $H$ is a 'subfield group'. Thus, $q_{0}=q_{1}^{m}$ with $m$ prime, and $H$ is the normalizer of $L\left(q_{1}\right)$. By [GMS01, 3.29, 3.30, 3.33] and the fact that $X / G$ has prime power order, it follows that $m \geq 3$ with equality only possible in the case that $T=\operatorname{Sz}\left(2^{a}\right)$ with $a$ odd and divisible by 3 . Write $\sigma$ as $\sigma=d f \tau$, where $d, f, \tau$ are suitable diagonal, field and graph automorphisms (possibly trivial). Since $\sigma$ is a $p$-element, we may take these also to be $p$-elements. Note that $\tau$ nontrivial implies that $p \leq 3$ with equality only possible when $T$ is an 8 -dimensional orthogonal group.

It follows from the arguments in [GMS01, 3.8] that $m=p$. We sketch a proof. As noted in GMS01, if $C$ is any $X$-stable conjugacy class of $G$, then $g \in C$ implies that $g$ has a fixed point on $\Omega$ (because then $X=G \mathbf{C}_{X}(g)$ and so $g \in \mathbf{C}_{X}\left(\sigma g^{\prime}\right)$ for some $g^{\prime} \in G$ and $\sigma g^{\prime}$ has a unique fixed point). We can take $C$ to be any $f \tau$ invariant class of semisimple elements (as such classes are invariant under diagonal automorphisms). Since $H$ does not contain elements from every class of semisimple elements, it follows that $f \tau \neq 1$.

Consider first the case where $f$ is not 1 , that is, a field automorphism is involved in $\sigma$. The centralizer of $f \tau$ will be a group $L\left(q_{2}\right)$ of the same type as $T$, possibly twisted with $q_{0}=q_{2}^{b}$ with $b$ a power of $p$. We see that if $m \neq p$, then there exist semisimple classes $C$ which intersect this $L\left(q_{2}\right)$ but do not intersect $L\left(q_{1}\right)$ (for example, we can take suitable elements whose orders are certain primitive prime divisors). This shows that $m=p$ in this case.

Next consider the case where $f=1$. Since $f \tau \neq 1, \tau$ is then nontrivial. It is easy to dispose of this case too. For example, if $L$ is linear of even dimension $n$, there exist semisimple classes in $\operatorname{PSp}_{n}\left(q_{0}\right)$ which do not meet $L_{n}\left(q_{1}\right)$.

We next show that $p$ does not divide the order of $T$. Since $\sigma$ has a unique fixed point, $|\Omega|$ has order prime to $p$, and so $H$ contains a Sylow $p$-subgroup of $X$, and hence $H \cap T$ contains a Sylow $p$-subgroup of $T$. In particular, $p$ does not divide $q_{0}$. Now we prove that $p$ does not divide $|H \cap T|$. For suppose it did. Since the order of $T$ is a power of $q_{0}$ times a product of cyclotomic polynomials evaluated at $q_{0}$ and the order of $H \cap T$ is the same with $q_{0}$ replaced by $q_{1}$, it follows (using the fact that $m=p$ ) that $p$ divides $|T: H \cap T|=|\Omega|$, which is a contradiction. Thus $p$ does not divide $|H \cap T|$, and since $H \cap T$ contains a Sylow $p$-subgroup of $T$, in fact $p$ does not divide $|T|$.

In particular, there are no nontrivial diagonal automorphisms of $T$ of order $p$. It follows that $\langle\sigma G\rangle$ and $\langle f G\rangle$ are both Sylow $p$-subgroups of the subgroup $\langle d G, f G\rangle$ of Aut $(T) / G$, and hence are conjugate in $\langle d G, f G\rangle$, that is, there exists an element $\tau \in\langle G, d, f\rangle$ such that $\left\langle(\sigma G)^{\tau}\right\rangle=\langle f G\rangle$. Now

$$
X^{\tau}=\left\langle\sigma^{\tau}, G^{\tau}\right\rangle=\left\langle f, G^{\tau}\right\rangle,
$$

and $\left(G^{\tau}, X^{\tau}, \Omega^{\prime}\right)$ is an exceptional triple equivalent to $(G, X, \Omega)$, where $\Omega^{\prime}=\left[X^{\tau}\right.$ : $\left.X_{\omega}^{\tau}\right]$. Thus Theorem 1.1(i) holds. 
By [GMS01, 3.29, 3.30], the only possible cases that are left with $X / G$ a $p$-group are:

(1) $T=\mathrm{PSL}_{2}\left(2^{e}\right)$ with $e$ odd, $p$ is odd, and $H \cap T$ dihedral of order $2\left(2^{e}+1\right)$;

(2) $T=\mathrm{PSL}_{2}\left(3^{e}\right)$ with $e>1$ odd and $H \cap T$ dihedral of order $3^{e}+1$;

(3) $T=\mathrm{PSL}_{2}\left(r^{e}\right), r>2$ with $e$ even, $p=2$, and $H \cap T$ dihedral of order $r^{e}-1$;

(4) $T=\operatorname{PSU}_{3}\left(2^{e}\right)$ with $e>1$ odd, $p=3$ and $H \cap T$ the stabilizer of an orthogonal decomposition of the natural module; or

(5) $T=\mathrm{Sz}\left(2^{e}\right), e$ a power of the odd prime $p \neq 5$, and $H \cap T$ is the normalizer of a Sylow 5-subgroup.

In the first case, we claim that $e$ is a power of $p$ and $\sigma$ is a field automorphism of order $e$. Clearly, $\sigma$ may be taken to be a field automorphism. If the order of $\sigma$ is $f$, then its centralizer is $\operatorname{PSL}_{2}\left(2^{e / f}\right)$, which must be contained in our dihedral group, whence $e=f$. Thus, $e$ is a power of $p$ and Theorem 1.1(ii) holds. By [FGS93] (see also [GMS01, 3.27]), there are examples in this case.

The second case turns out to be impossible here: If $p$ is odd, then this is not exceptional, since the centralizer of a field automorphism is not contained in $H$. If $p=2$, then $\sigma$ induces a diagonal automorphism and the centralizer of $\sigma t$ for various $t$ contains every possible semisimple element of $T$, which is a contradiction.

Next consider $T=\mathrm{PSL}_{2}\left(r^{e}\right), r>2$ with $e$ even and $H \cap T$ dihedral of order $r^{e}-1$. If $p \neq 2$, then $\operatorname{PSL}_{2}\left(r^{2}\right) \leq H$, which implies that $H$ is a subfield group. So $p=2$, as in Theorem 1.1(iii). This is an example by [GMS01, 3.28].

Next, consider $T=\mathrm{PSU}_{3}\left(2^{e}\right)$ with $e$ odd, $e>1$, and $H \cap T$ the stabilizer of an orthogonal decomposition of the underlying vector space. We claim that $\sigma$ must involve a diagonal automorphism. Otherwise for some $t \in T, \sigma t$ would be a field automorphism whose centralizer contains $\mathrm{PSU}_{3}(2)$. This subgroup does not act imprimitively and so is not contained in a conjugate of $H$. Since the group of outer diagonal automorphisms has order divisible by 3 , it follows that $p=3$. We claim that $e$ is a power of 3 . If not, choose $t \in T$ such that the centralizer of $\sigma t$ contains $\mathrm{SU}_{2}\left(2^{f}\right)$ with $f$ the $3^{\prime}$-part of $e$. If $f>1$, then this subgroup cannot embed in $H$. We have now shown that $X / G$ must be generated by the product of a diagonal automorphism and a field automorphism of order $e$. Thus Theorem 1.1(iv) holds. If $e$ is a power of 3, we have an example by [GMS01, 3.30].

Finally, the case $T=\mathrm{Sz}\left(2^{e}\right)$ appears in the conclusion of the theorem.

\section{Proof of Theorem 1.2}

In this section, we prove Theorem 1.2. First we determine the possible O'NanScott types of point-primitive TODs.

Lemma 4.1. Let $(G, X, \Omega, \mathcal{P})$ be a cyclic TOD with $X$ primitive on $\Omega$ such that $X / G$ is a cyclic p-group for some prime $p$. Then $X^{\Omega}$ is of affine, almost simple, simple diagonal, compound diagonal, or product action type. Moreover, for each of these types, there do exist examples.

Proof. It follows from Lemma 2.3 and the O'Nan-Scott Theorem that $X$ is of affine, almost simple, simple diagonal, compound diagonal, or product action type. We note that $p$ is coprime to $|\Omega|$, see [LP01, Lemma 2.5]. Thus, if $X$ is affine, then the socle of $X$ has order coprime to $p$. It is known that there exist examples of affine type; see for example [LP01, Section 6] for a quite general construction. By Theorem 1.1, there exist TODs of almost simple type; while by Constructions [2.5] 
2.8 and 2.9, there exist TODs of simple diagonal, compound diagonal, and product action types, respectively.

Remark 4.2. If $X^{\Omega}$ is of affine type, then $\operatorname{soc}(X)=\mathbb{Z}_{r}^{d}$ for some prime $r$, and as noted in the proof above, $r \neq p$.

Thus to complete the proof of Theorem 1.2 we need to verify the assertions in parts (iii), (iv), (v). First we deal with part (iii), namely cyclic TODs $(G, X, \Omega, \mathcal{P})$ such that $X$ is quasiprimitive of simple diagonal type. For a quasiprimitive permutation group $X$ with socle $G=T^{l}$ on $\Omega$, if $X$ is of simple diagonal type, then $G \leq X \leq G\left(\operatorname{Aut}(T) \times \mathrm{S}_{l}\right)$, where $\operatorname{Aut}(T) \cap G=\operatorname{lnn}(T)$ is identified with $H=$ $\{(t, \ldots, t) \mid t \in T\}$. Let $\omega \in \Omega$. Then we may identify $\Omega=[G: H]$ and $H=G_{\omega}$. For a $\operatorname{TOD}(G, X, \Omega, \mathcal{P})$ with $X=\langle G, \sigma\rangle$, we may take $\sigma \in X_{\omega} \leq \operatorname{Aut}(T) \times \mathrm{S}_{l}$ and $\sigma \notin G_{\omega}$.

Lemma 4.3. Let $X$ be a quasiprimitive permutation group on a set $\Omega$ of simple diagonal type with socle $G=T^{l}$ for some $l \geq 2$. Then there exists a partition $\mathcal{P}$ of $\operatorname{Orbl}(G, \Omega)$ such that $(G, X, \Omega, \mathcal{P})$ is a cyclic TOD of $p$-power index with p prime if and only if

(i) $l=p^{e}$ for some $e \geq 1$,

(ii) $p$ is coprime to $|T|$, and

(iii) there exists $\sigma \in X$ of the form $\sigma=\left(12 \ldots p^{e}\right) \in \mathrm{S}_{p^{e}}$.

Moreover, $(G, H, \sigma)$ is as in Construction [2.5, where $H=\{(t, \ldots, t) \mid t \in T\}$.

Proof. Let $H=\{(t, \ldots, t) \mid t \in T\}, \Omega=[G: H]$, and $\omega \in \Omega$ be such that $H=G_{\omega}$. Assume that conditions (i)-(iii) of the lemma are satisfied. By Lemma 2.6 $\sigma$ fixes no $H g H$ for $g \in G \backslash H$. Then by Construction 2.2 there exists a partition $\mathcal{P}$ of $\operatorname{Orbl}(G, \Omega)$ such that $(G, X, \Omega, \mathcal{P})$ is a TOD of $p$-power index.

Conversely, assume that $(G, X, \Omega, \mathcal{P})$ is a cyclic TOD of index $k$, where $k$ is a power of a prime $p$. Then by Lemma 2.1, there exists $\sigma \in X_{\omega}$ which has $p$-power order and fixes no element in $\operatorname{Orbl}(G, \Omega)$. Since $p$ divides $|\Omega|-1$, we have that $p$ is coprime to $|\Omega|=|T|^{l-1}$, and so $p$ is coprime to $|T|$; so part (ii) holds.

Consider the subgroup $Y:=\langle G, \sigma\rangle$. Then $(G, Y, \Omega, \mathcal{P})$ is also a cyclic TOD of index $k$. Suppose that $\langle\sigma\rangle$ is not transitive on $\left\{T_{1}, T_{2}, \ldots, T_{l}\right\}$. Then we may write $G=L \times N$ such that $L$ is a proper normal subgroup of $G$ and is normalized by $\sigma$, so that $L \triangleleft Y$. By Lemma 2.3 $L$ is intransitive on $\Omega$. Then since $L \triangleleft Y$, all $L$-orbits in $\Omega$ have the same length, and since $L \cap H=1$ it follows that $L$ is semiregular on $\Omega$. Let $B$ be an orbit of $L$ in $\Omega$. Then $L^{B} \cong L$ is a regular normal subgroup of $Y_{B}^{B}$, and by [LP01, Lemma 4.1], there exists a cyclic TOD $\left(G_{B}^{B}, Y_{B}^{B}, B, \mathcal{P}_{B}\right)$ of index $k$, which is a contradiction to Lemma 2.3. Therefore, $\langle\sigma\rangle$ is transitive on $\left\{T_{1}, T_{2}, \ldots, T_{l}\right\}$, and in particular, $l=p^{e}$ for some $e \geq 1$. Thus part (i) holds.

Recall that $Y=\langle G, \sigma\rangle \leq X<G$. $\left(\operatorname{Out}(T) \times \mathrm{S}_{p^{e}}\right)<\operatorname{Sym}(\Omega)$, and $\sigma \in X_{\omega}<$ Aut $(T) \times \mathrm{S}_{l}$. Then renumbering the $T_{i}$ if necessary, we may write $\sigma=\varphi\left(12 \ldots p^{e}\right)$, where $\varphi \in \operatorname{Aut}(T)$ and $\left(12 \ldots p^{e}\right) \in \mathrm{S}_{p^{e}}$. Suppose $\varphi \neq 1$. Since $\sigma$ has $p$-power order, also $\varphi$ has $p$-power order. We claim that $\mathbf{C}_{T}(\varphi)<\mathbf{C}_{T}\left(\varphi^{p^{e}}\right)$. If $\varphi^{p^{e}}=1$, then $\mathbf{C}_{T}(\varphi)<T=\mathbf{C}_{T}\left(\varphi^{p^{e}}\right)$, so the claim is true in this case. Thus we may assume that $\varphi^{p^{e}} \neq 1$. Then $p^{2}$ divides $o(\varphi)$. Since $p$ is coprime to $|T|$, we have that $p$ is odd and $\varphi \notin \operatorname{lnn}(T)$. It follows that $T$ is a simple group of Lie type. Further, it is easily shown that $p$ divides neither the order of a graph automorphism nor the order of a diagonal automorphism of $T$. It follows that $\varphi$ is a field automorphism 
of $T$. It then follows from [GO p. 102] that $\mathbf{C}_{T}(\varphi)<\mathbf{C}_{T}\left(\varphi^{p^{e}}\right)$, as claimed. Let $t \in \mathbf{C}_{T}\left(\varphi^{p^{e}}\right) \backslash \mathbf{C}_{T}(\varphi)$, and let $g=\left(t, t^{\varphi}, \ldots, t^{\varphi^{p^{e}-1}}\right) \in G$. Since $t^{\varphi} \neq t$, we have that $g \notin G_{\omega}$, so that $G_{\omega} g G_{\omega}$ is a non-trivial double coset; further, $g^{\sigma}=g$ since $t^{\varphi^{p^{e}}}=t$. Therefore, $\sigma$ leaves invariant the double coset $G_{\omega} g G_{\omega}$, and hence fixes the corresponding orbital, which is a contradiction. So $\varphi=1$ and part (iii) holds. Moreover, $(G, H, \sigma)$ are as in Construction 2.5

Remark. For each odd prime $p$, at least one of $\mathrm{A}_{5}, \mathrm{Sz}(8)$ and $\mathrm{PSL}_{2}(7)$ has order coprime to $p$. Then Construction 2.5 tells us that there exist TODs of index $p$ of simple diagonal type for each odd prime $p$.

By Lemma 4.3, the assertions of Theorem 1.2(iii) hold for the simple diagonal case. Now we consider point-primitive cyclic TODs of product action type and compound diagonal type. For convenience, we define some notation, which will be used throughout the rest of this section.

Notation 4.4. Let $X$ be a transitive permutation group on $\Omega$ such that $G:=$ $\operatorname{soc}(X)=N_{1} \times \cdots \times N_{l}$, where $l \geq 2$ and $N_{i} \cong N$ is nonabelian. Let $\mathcal{N}=$ $\left\{N_{1}, N_{2}, \ldots, N_{l}\right\}$, and assume that $X$ acts transitively on $\mathcal{N}$ by conjugation. Assume further that $\Omega=\Delta^{l}$ and $X \leq \operatorname{Sym}(\Delta) \gtrless \mathrm{S}_{l}$ in product action. Moreover, suppose that $N$ is a transitive subgroup of $\operatorname{Sym}(\Delta)$, so $G$ is transitive on $\Omega$. Let $\omega=(\delta, \ldots, \delta) \in \Omega$ with $\delta \in \Delta$, so that $G_{\omega}=H_{1} \times H_{2} \times \cdots \times H_{l}=H^{l}$, where $H=N_{\delta}$. For a subgroup $M$ of $X$, let $\Omega_{M}$ be the set of $M$-orbits in $\Omega$. Let $k=p^{e}$ with $p$ prime and $e \geq 1$.

We first treat a special case.

Lemma 4.5. Suppose that Notation 4.4 holds, and that $(G, Y, \Omega, \mathcal{P})$ is a cyclic TOD of index $k$ such that $Y=\langle G, \sigma\rangle \leq X$ for some $\sigma \in Y_{\omega}$. Assume also that $\langle\sigma\rangle$ acts transitively on $\left\{N_{1}, \ldots, N_{l^{\prime}}\right\}$ by conjugation for some $l^{\prime} \leq l$. Then $\sigma^{l^{\prime}}$ normalizes $N_{i}$ for each $i \leq l^{\prime}$, and for any $\left\langle N_{i}, \sigma^{l^{\prime}}\right\rangle$-invariant partition $\Delta^{\prime}$ of $\Delta$, there exists a partition $\mathcal{Q}^{\prime}$ of $\operatorname{Orbl}\left(N_{i}, \Delta^{\prime}\right)$ such that $\left(N_{i},\left\langle N_{i}, \sigma_{0}\right\rangle, \Delta^{\prime}, \mathcal{Q}^{\prime}\right)$ is a cyclic TOD of index $k^{\prime}$, where $k^{\prime}=p^{e^{\prime}} \geq p$, and $\sigma_{0}$ is the permutation of $\Delta^{\prime}$ induced by $\sigma^{l^{\prime}}$.

Proof. Since $\langle\sigma\rangle$ is transitive on $\mathcal{P}$, it follows that $k=p^{e}$ divides $o(\sigma)$, and so, replacing $\sigma$ if necessary by some power $\sigma^{j}$, we may assume that $\sigma$ is a $p$-element.

(1). Assume first that $l^{\prime}=l$. By Lemma 2.1 $\sigma$ fixes no $G_{\omega} g G_{\omega}$ for $g \in G \backslash G_{\omega}$. Since $\langle\sigma\rangle$ acts transitively on $\mathcal{N}$ and since $\sigma$ normalizes $G_{\omega}$, it follows that $\sigma^{l}$ normalizes each $N_{i}$ and each $H_{i}$. Hence we have $\sigma^{l}=\left(\sigma_{1}, \ldots, \sigma_{l}\right) \in \operatorname{Sym}(\Delta)^{l}$. By Lemma 2.7 $\sigma^{l}$ fixes no $H_{i} t H_{i}$ for $t \in N_{i} \backslash H_{i}$. Then by Lemma 2.1 there is a partition $\mathcal{Q}$ of $\operatorname{Orbl}\left(N_{i}, \Delta\right)$ such that $\left(N_{i},\left\langle N_{i}, \sigma_{i}\right\rangle, \Delta, \mathcal{Q}\right)$ is a cyclic TOD of index $k^{\prime}>1$, where $k^{\prime}$ divides $o\left(\sigma_{i}\right)$, which in turn divides $o\left(\sigma^{l}\right)$. Thus $k^{\prime}=$ $p^{e^{\prime}}$ for some positive integer $e^{\prime}$. Let $\Delta^{\prime}$ be an $\left\langle N_{i}, \sigma_{i}\right\rangle$-invariant partition of $\Delta$. Then by [LP01] Theorem 1.2], there exists a partition $\mathcal{Q}^{\prime}$ of $\operatorname{Orbl}\left(N_{i}, \Delta^{\prime}\right)$ such that $\left(N_{i},\left\langle N_{i}, \bar{\sigma}_{i}\right\rangle, \Delta^{\prime}, \mathcal{Q}^{\prime}\right)$ is a cyclic TOD of index $k^{\prime}$, where $\bar{\sigma}_{i}$ is the permutation of $\Delta^{\prime}$ induced by $\sigma_{i}$ and hence induced by $\sigma^{l}$. So $\bar{\sigma}_{i}=\sigma_{0}$, and the lemma holds.

(2). Now assume that $l^{\prime}<l$. Then, renumbering the $N_{i}$ if necessary, we may write $G=L \times M$ such that $L=N_{1} \times \cdots \times N_{l^{\prime}}, M=N_{l^{\prime}+1} \times \cdots \times N_{l}$, and both $L$ and $M$ are invariant under $\sigma$. Then $L, M$ are normal in $Y$, and $\sigma^{l^{\prime}}$ normalizes $N_{i}$ for all $i \leq l^{\prime}$. Consider the action of $Y$ on the set $\Omega_{M}$ of $M$-orbits. Since $X$ acts on $\Omega$ 
in product action and since $N$ is transitive on $\Delta$, the $M$-orbit containing the point $\left(\delta_{1}, \ldots, \delta_{l}\right)$ consists of all the points $\left(\gamma_{1}, \ldots, \gamma_{l}\right)$ with $\left(\gamma_{1}, \ldots, \gamma_{l^{\prime}}\right)=\left(\delta_{1}, \ldots, \delta_{l^{\prime}}\right)$. Thus each $M$-orbit corresponds to a (unique) point of $\Delta^{l^{\prime}}$. Hence the permutation group $\bar{Y}$ induced by $Y$ on $\Omega_{M}$ is a subgroup of $\operatorname{Sym}(\Delta)$ \& $\mathrm{S}_{l^{\prime}}$ in product action, and we may identify $\Omega_{M}$ with $\Delta^{l^{\prime}}$. Since $G=L \times M$, the permutation group $\bar{G}$ induced by $G$ on $\Delta^{l^{\prime}}$ is $\bar{G}=L=N_{1} \times \cdots \times N_{l^{\prime}}$, and since $Y=\langle G, \sigma\rangle$ we have $\bar{Y}=\langle\bar{G}, \bar{\sigma}\rangle$, where $\bar{\sigma}$ is the permutation of $\Delta^{l^{\prime}}$ induced by $\sigma$. Note that $\langle\bar{\sigma}\rangle$ is transitive on $\left\{N_{1}, \ldots, N_{l^{\prime}}\right\}$. By [LP01, Theorem 1.2], there exists a partition $\mathcal{P}^{\prime}$ of $\operatorname{Orbl}\left(\bar{G}, \Delta^{l^{\prime}}\right)$ such that $\left(\bar{G}, \bar{Y}, \Delta^{l^{\prime}}, \mathcal{P}^{\prime}\right)$ is a cyclic TOD of index $k$. If $l^{\prime}=1$, then $\left(\bar{G}, \bar{Y}, \Delta^{l^{\prime}}, \mathcal{P}^{\prime}\right)=\left(N_{1},\left\langle N_{1}, \sigma\right\rangle, \Delta, \mathcal{Q}\right)$, and $\Delta^{\prime}$ is an $\left\langle N_{1}, \sigma\right\rangle$-invariant partition of $\Delta$. Then by [LP01 Theorem 1.2], there exists a partition $\mathcal{Q}^{\prime}$ of $\operatorname{Orbl}\left(N_{1}, \Delta^{\prime}\right)$ such that $\left(N_{1},\left\langle N_{1}, \sigma_{0}\right\rangle, \Delta^{\prime}, \mathcal{Q}^{\prime}\right)$ is a TOD of index $k^{\prime}=k$, as required. If $l^{\prime} \geq 2$, we may apply part (1) of the proof to the TOD $\left(\bar{G}, \bar{Y}, \Delta^{l^{\prime}}, \mathcal{P}^{\prime}\right)$. Thus the lemma is proved.

If $X \leq \operatorname{Sym}(\Delta) \imath \mathrm{S}_{l}$ in product action on $\Delta^{l}$ and $X$ induces a transitive subgroup of $\mathrm{S}_{l}$, then $X_{1}=\mathbf{N}_{X}\left(N_{1}\right)$ has index $l$ in $X$ and is contained in $\operatorname{Sym}(\Delta) 2\left(1 \times \mathrm{S}_{l-1}\right)=$ $\operatorname{Sym}(\Delta) \times\left(\operatorname{Sym}(\Delta)<\mathrm{S}_{l-1}\right)$. Let $\rho$ be the natural projection map to $\operatorname{Sym}(\Delta)$. Then $\rho(X)$ is called the component of $X$. In particular, if $X$ is a primitive permutation group on $\Delta^{l}$ of product action type, then $\Delta$ and $\rho$ can be chosen so that $\rho(X)$ is an almost simple primitive group on $\Delta$; while if $X$ is a primitive permutation group on $\Delta^{l}$ of compound diagonal type, then $\Delta$ and $\rho$ can be chosen so that $\rho(X)$ is a primitive group on $\Delta$ of simple diagonal type.

The next lemma deals with the case where $X$ is primitive of product action type.

Lemma 4.6. Suppose that Notation 4.4 holds and that $G \leq F \leq X$ and $\mathcal{P}$ are such that $(F, X, \Omega, \mathcal{P})$ is a cyclic TOD of index $k$ and $X=\langle F, \sigma\rangle$ for some $\sigma \in X_{\omega}$. Assume that $X$ is primitive on $\Omega=\Delta^{l}$ of product action type. Then $N_{1}=T$ is nonabelian simple, and there exist an integer $l^{\prime} \leq l$, a partition $\Delta^{\prime}$ of $\Delta$ and a partition $\mathcal{Q}$ of $\operatorname{Orbl}\left(T, \Delta^{\prime}\right)$ such that

(i) $\left\langle T, \sigma_{0}\right\rangle$ is an almost simple primitive group on $\Delta^{\prime}$, where $\sigma_{0}$ is the permutation of $\Delta^{\prime}$ induced by $\sigma^{l^{\prime}}$, and

(ii) $\left(T,\left\langle T, \sigma_{0}\right\rangle, \Delta^{\prime}, \mathcal{Q}\right)$ is a cyclic TOD of index $k^{\prime}$, where $k^{\prime}=p^{e^{\prime}} \geq p$.

Proof. Since $X$ is primitive, $G=\operatorname{soc}(X)$ is transitive on $\Omega$, and by Lemma [2.1] $\sigma$ fixes no double coset $F_{\omega} g F_{\omega}$ where $g \in F \backslash F_{\omega}$, and hence $\sigma$ fixes no $G_{\omega} g G_{\omega}$ for $g \in G \backslash G_{\omega}$. By [LP01, Lemma 6.1], $k$ divides the size of each $\langle\sigma\rangle$-orbit in $\operatorname{Orbl}(F, \Omega)$. This implies that $k$ divides the size of each $\langle\sigma\rangle$-orbit in $\operatorname{Orbl}(G, \Omega)$. Then applying [LP01, Lemma 6.1] again, $\left(G,\langle G, \sigma\rangle, \Omega, \mathcal{P}^{\prime}\right)$ is a cyclic TOD of index $k$ for some partition $\mathcal{P}^{\prime}$ of $\operatorname{Orbl}(G, \Omega)$.

Assume that $\langle\sigma\rangle$ is transitive on $\left\{N_{1}, N_{2}, \ldots, N_{l^{\prime}}\right\}$, where $l^{\prime} \leq l$. Now $N_{i}=T$ is a nonabelian simple group and is transitive on $\Delta$. Let $\sigma^{l^{\prime}}=\left(\sigma_{1}, \ldots, \sigma_{l}\right) \pi$, where $\sigma_{i} \in \operatorname{Sym}(\Delta), \pi \in \mathrm{S}_{l}$, and $\pi$ fixes each $i \leq l^{\prime}$. Then for $i \leq l^{\prime}$ the group induced by $\left\langle G, \sigma^{l^{\prime}}\right\rangle$ on the $i^{\text {th }}$ copy of $\Delta$ is $\left\langle T, \sigma_{i}\right\rangle$. By the definition of product action type, the component of $X$ on this copy of $\Delta$ is a primitive almost simple group with socle $T$. Thus $T \leq\left\langle T, \sigma_{i}\right\rangle \leq \operatorname{Aut}(T)$. Let $\Delta^{\prime}$ be a $\left\langle T, \sigma_{i}\right\rangle$-invariant partition of $\Delta$ such that $\left\langle T, \sigma_{i}\right\rangle$ acts primitively on $\Delta^{\prime}$. Then $\left\langle T, \sigma_{i}\right\rangle$ acts faithfully on $\Delta^{\prime}$ as the almost simple group $\left\langle T, \sigma_{0}\right\rangle$, where $\sigma_{0}$ is the permutation of $\Delta^{\prime}$ induced by $\sigma_{i}$, and hence by $\sigma^{l^{\prime}}$. Then by Lemma 4.5 there exists a partition $\mathcal{Q}$ of $\operatorname{Orbl}\left(T, \Delta^{\prime}\right)$ such that $\left(T,\left\langle T, \sigma_{0}\right\rangle, \Delta^{\prime}, \mathcal{Q}\right)$ is a cyclic TOD of index $k^{\prime}$, where $k^{\prime}=p^{e^{\prime}} \geq p$. 
By Lemma 4.6, the assertions of Theorem 1.2(iv) hold for the case where $X^{\Omega}$ is of product action type. In order to complete the proof of Theorem 1.2 we need to prove the assertions about the compound diagonal case. This is treated in the next lemma.

Lemma 4.7. Suppose that Notation 4.4 holds and that $G \leq F \leq X$ and $\mathcal{P}$ are such that $(F, X, \Omega, \mathcal{P})$ is a cyclic TOD of index $k$ and $X=\langle F, \sigma\rangle$ for some $\sigma \in X_{\omega}$. Assume that $X$ is primitive on $\Omega=\Delta^{l}$ of compound diagonal type. Then there exist an integer $l^{\prime} \leq l$, an $\left\langle N, \sigma^{l^{\prime}}\right\rangle$-invariant partition $\Delta^{\prime}$ of $\Delta$, and a partition $\mathcal{Q}$ of $\operatorname{Orbl}\left(N, \Delta^{\prime}\right)$ such that

(i) $\left\langle N, \sigma_{0}\right\rangle$ is quasiprimitive on $\Delta^{\prime}$ of simple diagonal type, where $\sigma_{0}$ is the permutation of $\Delta^{\prime}$ induced by $\sigma^{l^{\prime}}$, and

(ii) $\left(N,\left\langle N, \sigma_{0}\right\rangle, \Delta^{\prime}, \mathcal{Q}\right)$ is a cyclic TOD of index $k^{\prime}$, where $k^{\prime}=p^{e^{\prime}} \geq p$, and $\left(N, N_{\delta}, \sigma_{0}\right)$ is as in Construction 2.5 for some $\delta \in \Delta^{\prime}$.

Proof. As $X$ is primitive, $G=\operatorname{soc}(G)$ is transitive on $\Omega$, and by Lemma $2.1 \sigma$ fixes no double coset $F_{\omega} g F_{\omega}$ where $g \in F \backslash F_{\omega}$, and hence fixes no $G_{\omega} g G_{\omega}$ for $G \in G \backslash G_{\omega}$. As in the first paragraph of the proof of Lemma 4.5, we may assume that $\sigma$ is a $p$-element. Then by Lemma 2.1, $(G,\langle G, \sigma\rangle, \Omega, \mathcal{P})$ is a cyclic TOD of index $k_{0}>1$, where $k_{0}$ divides $o(\sigma)$, so $k_{0}=p^{e_{0}} \geq p$. Suppose that $\langle\sigma\rangle$ acts transitively on $\left\{N_{1}, N_{2}, \ldots, N_{l^{\prime}}\right\}$ by conjugation for some $l^{\prime} \leq l$. Then $\sigma^{l^{\prime}}$ normalizes $N=N_{i}$ for each $i \leq l^{\prime}$. By Lemma 4.5 there exists a partition $\mathcal{Q}$ of $\operatorname{Orbl}(N, \Delta)$ such that $\left(N,\left\langle N, \sigma_{0}\right\rangle, \Delta, \mathcal{Q}\right)$ is a cyclic TOD of index $k^{\prime}$, where $k^{\prime}=p^{e^{\prime}} \geq p$, and $\sigma_{0}$ is as stated in the lemma.

If $N$ is a minimal normal subgroup of $\left\langle N, \sigma_{0}\right\rangle$, then $\left\langle N, \sigma_{0}\right\rangle$ is quasiprimitive on $\Delta$ of simple diagonal type, and by Lemma 4.3 parts (i) and (ii) follow with $\Delta^{\prime}=\Delta$.

Assume now that $N$ is not a minimal normal subgroup of $\left\langle N, \sigma^{l^{\prime}}\right\rangle$. Then $N=$ $L \times M=T^{l_{0}}$ with $M$ a nontrivial normal subgroup and $L$ a minimal normal subgroup of $\left\langle N, \sigma^{l^{\prime}}\right\rangle$. By Lemma 2.3, we conclude that $l_{0} \geq 3$. Suppose first that $L$ is simple. Since $l_{0} \geq 3, L$ is intransitive on $\Delta$ and $M$ is a regular normal subgroup of $\left\langle N, \sigma_{0}\right\rangle$, which is a contradiction to Lemma 2.3 Thus $L$ is not simple. Similarly, $M$ is not simple. Therefore, $M$ is intransitive and $L$ is not regular on $\Delta$. Now the factor group $\left\langle N, \sigma_{0}\right\rangle / M$ induces a quasiprimitive permutation group on $\Delta_{M}$ of simple diagonal type. Let $\bar{\sigma}_{0}$ be the permutation of $\Delta_{M}$ induced by $\sigma_{0}$, and write $\bar{L}=N / M$ and $\Delta^{\prime}=\Delta_{M}$. Let $\bar{H}$ denote the stabilizer in $\bar{L}$ of an element of $\Delta^{\prime}$. Then by [LP01, Theorem 1.2], there exists a partition $\mathcal{Q}^{\prime}$ of $\operatorname{Orbl}\left(\bar{L}, \Delta^{\prime}\right)$ such that $\left(\bar{L},\left\langle\bar{L}, \bar{\sigma}_{0}\right\rangle, \Delta^{\prime}, \mathcal{Q}^{\prime}\right)$ is a cyclic TOD of index $k^{\prime}$, and by Lemma 4.3 the corresponding triple $\left(\bar{L}, \bar{H}, \Omega_{M}\right)$ is as in Construction 2.5

We end this section with a summary of the arguments for proving Theorem 1.2

Proof of Theorem 1.2. Let $(G, X, \Omega, \mathcal{P})$ be a cyclic TOD of index $p^{e}$, where $p$ is a prime and $e \geq 1$, such that $X$ is primitive on $\Omega$. By Lemma $4.1 X$ is of affine, almost simple, simple diagonal, compound diagonal, or product action type. By Remark 4.2 the assertions of Theorem[1.2(i) hold for the affine case, and those for the almost simple case in Theorem 1.2(ii) follow from Theorem 1.1. Finally the assertions for the simple diagonal, compound diagonal and product action types in Theorem 1.2(iii), (iv), (v) follow from Lemmas 4.3, 4.7, 4.6, respectively. 


\section{Proof of Theorem 1.3}

This section is devoted to proving Theorem 1.3, regarding vertex-transitive selfcomplementary graphs.

Proof of Theorem [1.3. Let $\Gamma$ be a vertex-transitive self-complementary digraph with vertex set $V$. Let $\sigma$ be a complementary permutation for $\Gamma$, that is, $\sigma \in$ $\operatorname{Sym}(V)$ and $\Gamma^{\sigma}=\bar{\Gamma}$. Let $G=$ Aut $\Gamma$, and let $X=\langle G, \sigma\rangle$. Let $E$ and $\bar{E}$ be the edge sets of $\Gamma$ and $\bar{\Gamma}$, respectively. Let $\mathcal{E}, \overline{\mathcal{E}}$ be the set of $G$-orbitals in $V$ whose union is $E, \bar{E}$ respectively, and let $\mathcal{P}=\{\mathcal{E}, \overline{\mathcal{E}}\}$. Then $(G, X, V, \mathcal{P})$ is a cyclic TOD of index 2. Assume that $X$ is primitive on $V$. Since $|V|$ is odd (see [LP01, Lemma 2.5]), by Theorem 1.2 $X$ is affine, almost simple, or of product action type. Moreover, if $X$ is affine, then $\operatorname{soc}(X)$ has odd order; if $X$ is almost simple, then by Lemma 3.1 $\operatorname{soc}(X)=\operatorname{PSL}_{2}\left(q^{2}\right)$ with $q$ a power of an odd prime; and if $X$ is of product action type, then $\operatorname{soc}(X)$ is a product of copies of $\operatorname{PSL}_{2}\left(q^{2}\right)$.

Thus, to complete the proof of Theorem 1.3 we only need to prove that in the case where $\operatorname{soc}(X)=\operatorname{PSL}_{2}\left(q^{2}\right), E$ must not be symmetric. Let $T=\operatorname{soc}(X)$. Then by Theorem 1.1, $H \cong \mathrm{D}_{q^{2}-1},|V|=q^{2}\left(q^{2}+1\right) / 2, G / T$ is of odd order, and $\sigma$ is an involutory field-diagonal automorphism of $G$.

Write $\sigma=f d$ with $d$ diagonal, in the split torus of $\operatorname{PGL}_{2}\left(q^{2}\right)$, and $f$ a field automorphism. We can think of $V$ here as the set of all involutions of $T$ with $X \leq$ $\mathrm{P} L_{2}\left(q^{2}\right)$ acting by conjugation. We shall produce a pair $(t, u)$ of involutions such that the orbital containing $(t, u)$ is mapped by $\sigma$ to the paired orbital containing $(u, t)$. To do this, choose involutions $t, u \in \mathrm{PGL}_{2}(q)=\mathbf{C}_{T}(f)$ with the product $t u$ having order $q-\epsilon$, where $q$ equals $\epsilon$ modulo 4 . Note that there is no $g \in T$ with $\left(t^{g}, u^{g}\right)=(u, t)$ : otherwise this $g$ inverts $t u$, so $g$ belongs to $\mathbf{N}_{T}(t u)$; however, $\left|\mathbf{N}_{T}(t u): \mathbf{N}_{\mathrm{PGL}_{2}(q)}(t u)\right|=(q+\epsilon) / 2$ is odd. So we may take $g \in \mathbf{N}_{\mathrm{PGL}_{2}(q)}(t u)=$ $\mathrm{D}_{2(q-\epsilon)}$. However $u$ and $t$ are not conjugate in $\mathrm{D}_{2(q-\epsilon)}$. Hence $t$ and $u$ are not conjugate in $\mathbf{N}_{T}(t u)$. On the other hand, they are conjugate in $\mathbf{N}_{\mathrm{PGL}_{2}\left(q^{2}\right)}(t u)$. It follows that $(u, t)^{T}=(t d, u d)^{T}=(t \sigma, u \sigma)^{T}=(t, u)^{T \sigma}$. Hence we have found a non-trivial orbital $(u, t)^{T}$ that is sent to its paired orbital $(t, u)^{T}$ by $\sigma$. Thus one of $E, \bar{E}$ contains $(u, t)^{T}$ and the other contains $(t, u)^{T}$. So $E$ is not symmetric. Hence in this case there is no TOD $(G, X, V, \mathcal{P})$ of index 2 with each of the two parts of $\mathcal{P}$ symmetric. This completes the proof.

Remark. The proof shows that in the case where $\operatorname{soc}(G)=\operatorname{PSL}_{2}\left(q^{2}\right), V$ may be identified with the set of involutions of $\operatorname{soc}(G)$ in such a way that $\langle G, \sigma\rangle$ acts by conjugation. Moreover, $\sigma=d f$, where $d$ is a diagonal automorphism in the split torus of $\mathrm{PGL}_{2}\left(q^{2}\right)$ and $f$ is a field automorphism such that $\mathbf{C}_{G}(f)=\mathrm{PGL}_{2}(q)$. Suppose that $t, u$ are involutions such that $o(t u)$ divides $q-\varepsilon($ where $q \equiv \varepsilon(\bmod 4))$ and $o(t u)>2$. Then it is shown in the proof that exactly one of the orbitals $(t, u)^{G}$ and $(u, t)^{G}$ lies in $E$. This means that for each pair of $G$-orbitals of this type exactly one of them can be chosen to lie in $E$, giving several possibilities for $\Gamma$. In fact, different $\mathrm{P} \Gamma \mathrm{L}\left(2, q^{2}\right)$-conjugacy classes of elements $t u$ give different pairs of orbitals, and hence there are at least $(q-3) /\left(4 \log _{p} q\right)$ such orbital pairs (where $q$ is a power of the prime $p)$, and hence at least $2^{(q-3) /\left(4 \log _{p} q\right)}$ self-complementary graphs admitting $G$.

An isomorphism $\varphi$ between two of these graphs, say $\Gamma$ and $\Gamma^{\prime}$, is a permutation of $V$, and $(\text { Aut } \Gamma)^{\varphi}=\operatorname{Aut} \Gamma^{\prime}$. Since $\operatorname{soc}(G)=\operatorname{soc}($ Aut $\Gamma)=\operatorname{soc}\left(\right.$ Aut $\left.\Gamma^{\prime}\right)$, we have $\varphi \in \mathbf{N}_{\operatorname{Sym}(V \Gamma)}(\operatorname{soc}(G)) \cong \operatorname{PLL}_{2}\left(q^{2}\right)$. The number of these graphs isomorphic to 
a given graph $\Gamma$ is therefore at most $\left|\mathrm{P}_{2}\left(q^{2}\right): \mathrm{PSL}_{2}\left(q^{2}\right)\right|=4 \log _{p} q$. Hence there are, up to isomorphism, at least $2^{(q-3) /\left(4 \log _{p} q\right)} /\left(4 \log _{p} q\right)$ vertex-transitive self-complementary graphs admitting $G$.

We remark that in contrast to Theorem 1.3 in a TOD $(G, X, V, \mathcal{P})$, if $X / G$ is cyclic of odd prime power order, it is easy to see that we can always choose a transitive orbital decomposition of $G$ so that all parts are symmetric.

The authors thank the referee for pointing out an error in an earlier version of the paper.

\section{REFERENCES}

[FKS81] B. Fein, W. M. Kantor, M. Schacher, Relative Brauer groups. II, J. Reine Angew. Math. 328 (1981), 39-57. MR 83a:12018

[FGS93] M. Fried, R. Guralnick, J. Saxl, Schur covers and Carlitz's conjecture, Israel J. Math. 82 (1993), 157-225. MR 94j:12007

[GO] D. Gorenstein, Finite Simple Groups, 1982, Plenum Press, New York. MR 84j:20002

[GL83] D. Gorenstein, R. Lyons, The local structure of finite groups of characteristic 2 type, Mem. Amer. Math. Soc. 42 (1983), no. 276. MR 84g:20025

[GMS01] R. M. Guralnick, P. Müller, J. Saxl, The rational function analogue of a question of Schur and exceptionality of permutation representations, Mem. Amer. Math. Soc. 162 (2003), no. 773.

[Li97] C. H. Li, On self-complementary vertex-transitive graphs, Comm. Algebra 25 (1997), 3903-3908. MR 98k:05119

[LP00] C. H. Li, C. E. Praeger, Self-complementary vertex-transitive graphs need not be Cayley graphs, Bull. London Math. Soc. 33 (2001), 653-661. MR 2002h:05085

[LP01] C. H. Li, C. E. Praeger, On partitioning the orbitals of a transitive permutation group, Trans. Amer. Math. Soc. 355 (2003), No. 2, 637-653.

[LPS00] M. Liebeck, C. E. Praeger and J. Saxl, Transitive subgroups of primitive permutation groups, J. Algebra 234 (2000), no. 2, 291-361. MR 2001i:20003

[Muz99] M. Muzychuk, On Sylow's subgraphs of vertex-transitive self-complementary graphs, Bull. London Math. Soc. 31 (1999), 531-533. MR 2000i:05093

[Sac62] H. Sachs, Über selbstcomplementäre Graphen, Publ. Math. Debrecen 9 (1962), 270-288. MR 27:1934

Department of Mathematics, University of Southern California, Los Angeles, CalIFORNIA 90089

E-mail address: guralnic@math.usc.edu

School of Mathematics and Statistics, The University of Western Australia, Crawley, Western Australia 6009, Australia

E-mail address: li@maths.uwa.edu.au

School of Mathematics and Statistics, The University of Western Australia, CrawLey, Western Australia 6009, Australia

E-mail address: praeger@maths.uwa.edu.au

Department of Pure Mathematics and Mathematical Statistics, Centre for Mathematical Sciences, University of Cambridge, Wilberforce Road, Cambridge CB3 0WB, ENGLAND

E-mail address: saxl@dpmms.cam.ac.uk 